\title{
Chemoprevention of metastasis
}

Editorial

\section{Nancy J. Emenaker, Enrique Zudaire, and Brad St. Croix \\ Comment on: $X \cup L$, et al. COX-2 inhibition potentiates antiangiogenic cancer therapy and prevents metastasis in preclinical mod-} els. Sci Transl Med. 2014 25; 6:242ra84

Cancer chemoprevention employs natural, synthetic, or biologic substances to reverse, suppress, or prevent the development of cancer. Initially these agents were developed to reduce cancer risk or retard its development, but were not intended to treat cancer. Celecoxib, a selective cyclooxygenase-2 (COX-2) inhibitor, is used clinically to prevent polyp formation in familial adenomatous polyposis (FAP) patients, a population at high risk for colorectal cancer development. However, recent preclinical studies suggest that chemopreventive agents like celecoxib may have effective anti-tumor and anti-metastatic properties against advanced stage disease. Currently, a randomized clinical trial is underway to determine if celecoxib can indeed enhance the therapeutic value of oxaliplatin, leucovorin and fluorouracil in the adjuvant setting of resected colorectal cancer (CALGB 80702; NCT01150045) [1].

Recent preclinical tumor studies suggest that the effectiveness of celecoxib against late stage colon and breast cancers can be significantly enhanced when combined with an anti-angiogenic agent (i.e., bevacizumab, neutralizing anti-VEGF antibody) or a receptor tyrosine kinase inhibitor (i.e., axitinib, selectively targeting VEGF receptors) [2] (Figure 1). COX-2 played a key regulatory role in prostaglandin $\mathrm{E}_{2}$ $\left(\mathrm{PGE}_{2}\right)$ biosynthesis, driving angiogenesis in CT26 VEGF inhibitor refractory colon tumors. Moreover, activation of the COX-2/PGE 2 pathway also enhanced myeloid cell recruitment into tumors likely increasing production of additional pro-angiogenic factors and further fueling angiogenesis. $\mathrm{PGE}_{2}$ has also been found to promote tumor cell growth, and prevent apoptosis and immune suppression. Thus, $\mathrm{PGE}_{2}$ likely promotes tumor growth through multiple mechanisms.

Because tumor vessels provide an escape route for tumor cells, we hypothesized that simultaneous targeting of the VEGF and COX-2/PGE 2 pathways may also aid in blocking metastases. Indeed, in both experimental and spontaneous colon and breast cancer models dual VEGF/ COX-2 pathway inhibition reduced metastasis [2]. For example, six months post axitinib/celecoxib therapy $60 \%$ of the mice were cured by the combination compared to only $8 \%$ (axitinib) or $17 \%$ (celecoxib) in the monotherapy arms. These studies highlight the potential advantage of combinatorial approaches using anti-angiogenic agents with the chemopreventive agent celecoxib for treating metastases.

Celecoxib is the only clinically approved chemoprevention agent used for high risk FAP patients. While potential cardiotoxicities prevent its chemopreventive use in the general population, as an adjuvant therapy for blocking recurrence of late stage disease the potential benefits may be worth the risk, at least for some patients. Indeed, re-analysis of six large randomized placebo-controlled clinical trials and 72 epidemiological studies found no evidence of celecoxib induced cardiotoxicity in patients with a low baseline cardiovascular risk [3].

As with other targeted agents, identifying patients most likely to respond to VEGF and/or COX-2 inhibition will be a key factor influencing the success of future clinical trials in this area. Appropriate patient stratification would help ensure maximal responses and prevent potential toxicities in individuals unlikely to respond. Unfortunately, in the angiogenesis field reliable, predictive bioassays for directly measuring the responsiveness of different tumor types to anti-angiogenic agents are not yet available. While it is also difficult to predict who

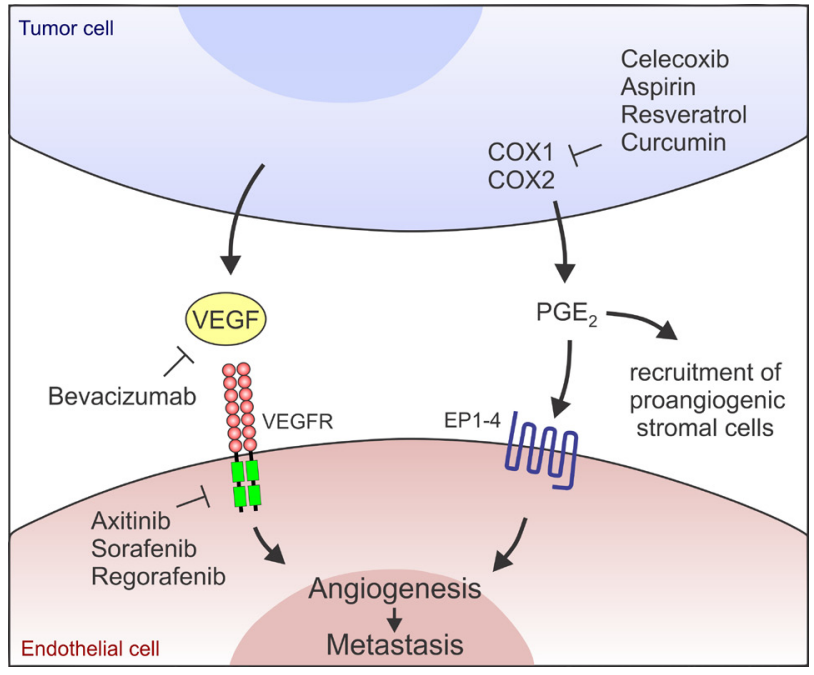

Figure 1: A model highlighting the independent nature of the VEGF/VEGFR and COX/PGE 2 pathways. Dual inhibition of the pathways at the indicated junctures may result in the most effective inhibition of angiogenesis and metastasis. 
will respond to COX-2 inhibition, some studies suggest greater benefit is obtained when tumors overexpress COX2. Mutations in $P I K 3 C A$ activate the phosphatidylinositol 3-kinase (PI3K) pathway and are present in approximately 15 to $20 \%$ of colorectal cancers, resulting in higher COX-2 expression and predicting colorectal cancer responsiveness to aspirin, a non-selective COX-1 and COX-2 inhibitor [4]. Mutations in the gene encoding 15-hydroxyprostaglandin dehydrogenase (15-PGDH), the enzyme responsible for $\mathrm{PGE}_{2}$ degradation, can also lead to high local $\mathrm{PGE}_{2}$ levels making colorectal cancer resistant to celecoxib therapy [5]. Thus, tumors which harbor activating $P I K 3 C A$ mutations and maintain wildtype 15 $P G D H$ may be the most likely to benefit from celecoxib or other non-steroidal anti-inflammatory drugs (NSAIDs).

What are the future possibilities for COX2 inhibition in individuals with an elevated risk for cardiovascular events? One possibility may be to use other less cardiotoxic NSAIDs like aspirin or naproxen. However, these NSAIDs also inhibit COX-1 imputing increased risks for gastrointestinal and renal toxicities. Another possibility may be to substitute COX inhibitors for other tumoricidal agents such as resveratrol, curcumin, vitamin D, naringenin or their synthetic derivatives, which are well tolerated and have been shown to block COX-2 expression. Although soluble epoxide hydrolase $(\mathrm{sEH})$ inhibitors used as a monotherapy may promote angiogenesis, when combined with celecoxib they also appear to potently suppress angiogenesis, tumor growth, and metastasis [6]. Similarly, COX-2/sEH dual pharmacological inhibitors have shown promising antitumor activity in preclinical studies [6]. Importantly, combination $\mathrm{COX}-2 / \mathrm{sEH}$ therapy may circumvent the cardiotoxicities associated with COX-2 inhibition by maintaining cardioprotective prostacyclin $\left(\mathrm{PGI}_{2}\right)$ to thromboxane $\mathrm{A}_{2}\left(\mathrm{TXA}_{2}\right)$ ratios. [6]. Therefore, clinically approved agents such as sorafenib and regorafenib, which inhibit VEGFRs and are also among the most potent sEH inhibitors ever identified [7], are promising agents to test in combination with celecoxib. Such combinatorial therapies could potentially help in the prevention or treatment of metastasis while minimizing cardiotoxicities.

Brad St. Croix: Tumor Angiogenesis Section, Mouse Cancer Genetics Program (MCGP), National Cancer Institute (NCI) at Frederick, NIH, Frederick, MD, USA

Correspondence: Brad St. Croix, email stcroix@ncifcrf.gov

Received: August 21, 2014;

Published: August 24, 2014;

\section{REFERENCES}

1. Chia WK et al. Nat Rev Clin Oncol 2012; 9: 561

2. Xu L et al. Sci Transl Med 2014; 6: 242ra84

3. Wang D et al. Annu Rev Med 2013; 64: 131

4. Liao X et al. N Engl J Med 2012; 367: 1596

5. Yan M et al. Proc Natl Acad Sci U S A 2009; 106: 9409

6. Zhang G et al. Proc Natl Acad Sci U S A 2014; 111: 11127

7. Hwang SH et al. Bioorg Med Chem Lett 2013; 23: 3732 